\title{
Software to Determine Optimal Oligonucleotide Sequences Based on Hybridization Simulation
}

\section{Data}

\author{
D. Hyndman ${ }^{1}$, A. Cooper ${ }^{1}$, S. Pruzinsky ${ }^{1}$, D. Coad $^{1}$ and M. Mitsuhashi ${ }^{1,2}$ \\ ${ }^{1}$ Advanced Gene Computing Technologies, Inc., Irvine, CA, and ${ }^{2}$ Hitachi Chemical Research Center, \\ Irvine, CA, USA
}

BioTechniques 20:1090-1097 (June 1996)

\begin{abstract}
In the design of oligonucleotide sequences for targeting DNA or RNA sequences, it can be difficult to identify sequences that will hybridize only to the intended target. The term "sequence-specific" or "sequence-nonspecific" is often used to describe the interactions of an oligonucleotide with a mixture of DNA or RNA. Our new computer program, HYBsimulator ${ }^{\mathrm{TM}}$ (formerly OligoProbe DesignStation), creates a set of candidate oligonucleotides from a target gene. For each of the candidate oligonucleotides, a large sequence database is searched for sequences that will hybridize to the oligonucleotide. This is referred to as computer hybridization simulation (CHS). Using the nearest-neighbor model, the HYBsimulator takes into account mismatches in hybridization and calculates the melting temperature $\left(T_{m}\right)$ or free energy for hybridization to all sequences in a database. The specificity of each oligonucleotide is then quantified by the number of genes that may hybridize and the predicted $T_{m}$ or free energies of hybridization to those genes. The CHS data are used to select oligonucleotides based on their specificity with respect to a database.
\end{abstract}

\section{INTRODUCTION}

Oligonucleotides are essential components for various types of biological experiments. They can be used for sequencing reactions, gene amplification procedures, specific gene detection (Northern/Southern blot experiments or in situ hybridization), detection of point mutations or the inhibition of gene expression with antisense oligonucleotides. Because they can be affordably synthesized, oligonucleotides are now frequently used in a wide range of basic and applied sciences, including the food, pharmaceutical and cosmetic industries. A major problem encountered when using oligonucleotides is the difficulty in obtaining specific sequences for each application. The terms "sequence-specificity" or "sequence-nonspecificity" are often used, but there is no consensus as to how to define and quantify sequence specificity. Previously $(14,15)$, we introduced a new concept for designing oligonucleotides based on a technique for calculating and representing specificity of oligonucleotide sequences. Using HYBsimulator $^{\mathrm{TM}}$, which runs on an $\mathrm{IBM}^{\circledR}$-compatible PC or Power Macintosh ${ }^{\circledR}$, we have adapted our technology for the design of oligonucleotides for many different applications. Here we discuss the capabilities and applications of HYBsimulator for the design of oligonucleotide sequences.

\section{LIMITATIONS OF CURRENTLY AVAILABLE SOFTWARE}

Programs for designing polymerase chain reaction (PCR) primer pairs are available from various commercial and noncommercial sources $(11,16)$. These programs select primer pairs that satisfy user-defined parameters, such as length, secondary structure of primers, G/C content of primers and amplicons, stability of primers, length of amplicons, etc. Because such software does not provide information regarding the specificity of the oligonucleotides, users cannot determine if particular primers will hybridize to other sequences in the genome. Another deficiency is that such software sometimes produces a long list of candidate primer pairs, making it arduous to initially select the "best" primers. These programs are also not suitable for antisense oligonucleotides.

Homology analysis programs $(2,10,12,17,19,23)$ and mul- 
tiple alignment programs $(5,9)$ are sometimes used to identify specific oligonucleotides. These programs compare the similarity of long sequences and are designed for identifying homologous genes in a database. Because they compare the entire sequence, they are not well-suited for identifying short specific oligonucleotide sequences (Figure 1, I). Also, if multiple copies of a short sequence are present within the same gene, a multiple alignment program will only identify one of those sequences, since the entire sequence is being compared (Figure 1, II). Furthermore, because some sequences in GenBank ${ }^{\circledR}$ contain introns (genomic DNA), and some sequences for the same gene may not contain introns (cDNA or RNA), a homology analysis often will not identify similar genes (Figure 1, III). Lastly, "homology" implies sequence similarity, which is not necessarily related to hybridization strength. If a sequence is found to be the most "homologous" to another sequence, it is not necessarily the sequence that is the most favorable for hybridization ("hybridizable") (Figure 1, IV).

Another way to estimate the specificity of particular sequences in the genome is to estimate the frequency of a given oligonucleotide based on the frequencies of the constituent dimer, trimer, tetramer, pentamer and hexamers of the oligonucleotide (7). While such a technique may identify oligonucleotides containing hexamers, which are present in the genome in high frequency, it cannot be expected to accurately predict the presence or absence of an actual 20-mer.

\section{PRINCIPLE OF HYBsimulator}

The principle of HYBsimulator is to simulate actual hybridization experiments between probes and sequences in a database, then select probes based on the data as outlined in Figure 2. Hybridization simulation requires three files: a model, a ProbeSet and a sequence library.

\section{Model}

HYBsimulator calculates melting temperature $\left(\mathrm{T}_{\mathrm{m}}\right)$ and free energy of hybridization using the nearest-neighbor model. The nearest-neighbor model calculates the total enthalpy or entropy of hybridization based on contributions of every

1. Difficulty in finding short oligonucleotide sequences. AGCT AGCATTAGCTAGCTAGGGTGCCATGGCAGG : : : : : $:$ : : : : $:$ : : : : : : : ACCTTTCAATGACTACCTAOCGAGCATTAGCGGG : : : : : : : : : : : : : $:$ : $:$ : : : ACCTAGCATTAGCTAGCTAGGGTTCCATGARGG

II. Difficulty in finding multiple repeats. ACCTAGCATTAGCTACCTACCGAGCATTAGCGGG : : : ::::::::::1:: : :: : : : ::: ACCTAGCATTAGCTAGCTAGGGTTCCATGARGGG

III. Interference of homology by introns. GGACGATGCAGGAAC $:::::::$ GGACGATAGCTTGAGATGCAGGAGGAAC

IV. Sequence similarity does not correlate with hybridization strength. AATTATTTTAAGCTGAACCTGCCCGCGGCACTTA

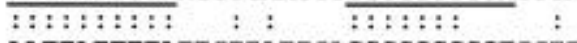
AATTATTTTATTGTTATTGAGCCCGCGCGTGGTT Homology $-100 \%$ Homology $=70 \%$ $\Delta \mathrm{G}=12.6 \mathrm{Kcal} / \mathrm{mol} \quad \Delta \mathrm{G}=17.8 \mathrm{Keal} / \mathrm{mol}$

Figure 1. Summary of current problems of homology/multiple alignment programs for designing optimal oligonucleotide sequences. two consecutive base pair combination. $\mathrm{T}_{\mathrm{m}}$ or free energy is determined from the total entropy and enthalpy $(3,6)$. The parameters for the contributions of the different nearest-neighbor combinations are contained in the model files. The parameters for DNA:DNA (3), RNA:RNA $(6,22)$ or RNA:DNA (21) are different and are specified by the appropriate model file. When new parameters become available (i.e., for phosphorothioate oligonucleotides or peptide nucleic acid), they can be put into a new model file for use by HYBsimulator. The model files also contain values that are used for calculating the $\mathrm{T}_{\mathrm{m}}$ or free energy for hybridization with mismatches $(1,8,18,20)$.

\section{ProbeSet}

A ProbeSet is a set of candidate oligonucleotides. A ProbeSet created by HYBsimulator contains all possible oligonucleotides derived from the target sequence that fit a chosen specification. ProbeSets can be created with a specified length, $\mathrm{T}_{\mathrm{m}}$ or free energy of hybridization. HYBsimulator creates a candidate oligonucleotide from each position in the target gene sequence that is closest to fitting the specified input criteria (i.e., $\mathrm{T}_{\mathrm{m}}$, free energy or length). For example, if the target gene is $1000 \mathrm{bp}$, approximately 1000 candidate oligonucleotides will be created and stored in the ProbeSet. Once a ProbeSet is created, the user can view the sequence of each oligonucleotide, edit the sequences, complement the entire ProbeSet or add a tag sequence to each oligonucleotide entry. Also, a ProbeSet can be created by inputting oligonucleotide sequences directly.

\section{Hybridization}

It is from a ProbeSet that the designed oligonucleotides are selected for a particular application. The specificity of probes in the ProbeSet is determined based on computer hybridization simulation (CHS) data. CHS simulates hybridization of each probe in the ProbeSet with every sequence in a specified GenBank database. For each sequence to which a particular probe may hybridize, the binding strength (as $\mathrm{T}_{\mathrm{m}}$ or free energy) is calculated using parameters provided by the selected

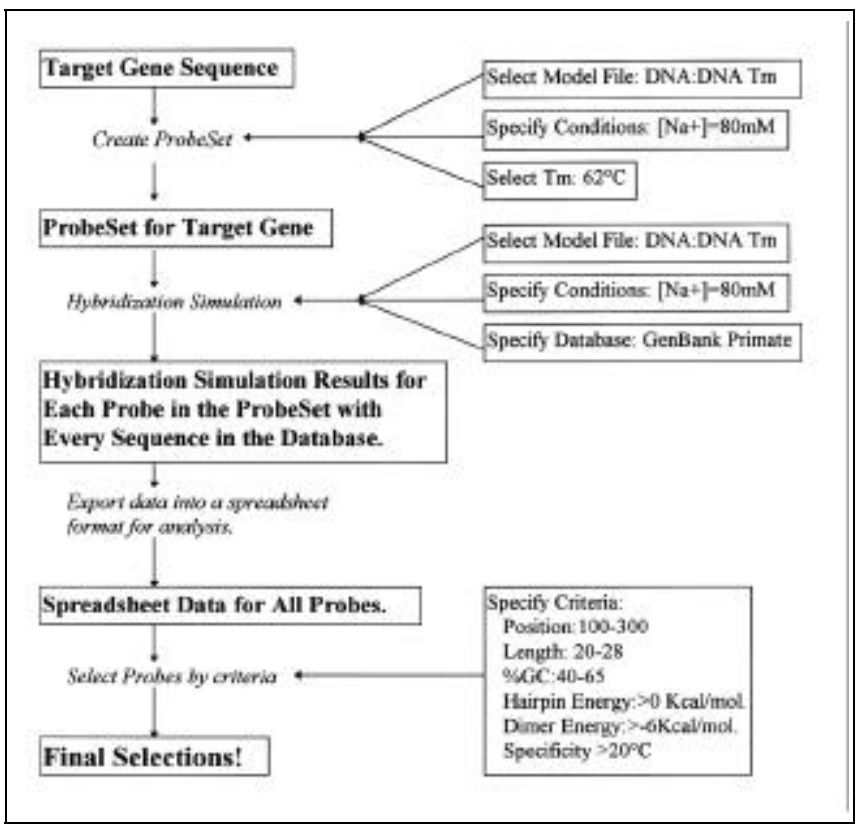

Figure 2. HYBsimulator strategy for design of oligonucleotides. 
model file. In addition to the conventional calculation of $\mathrm{T}_{\mathrm{m}}$ or free energy for the entire length of the probe, HYBsimulator performs multiple calculations for the possible sub-sequences, then selects the most favorable value, as shown in Figure 3, I. This calculation will yield the value for the most stable hybridization of a probe to a mismatched sequence (Figure 3, II). CHS for a 1000-bp gene with the GenBank primate database takes approximately $4 \mathrm{~h}$ with typical hardware (Pentium $^{\circledR}$ PC with 32 Mbytes random-access memory [RAM]).

For CHS, HYBsimulator accepts the GenBank Flat File CD-ROM (National Center for Biotechnology Information [NCBI], Bethesda, MD, USA). Also, HYBsimulator can use a researcher's own sequence database if the data are in the GenBank ASCII format.

\section{VIEWING AND ANALYSIS OF HYBsimulator RESULTS}

\section{Hybridization Window}

The Hybridization window in HYBsimulator displays CHS data (Figure 4). The left half of the window contains a graphical representation of all of the CHS data generated. The right half of the window displays specific data for individual probes. Each position on the $\mathrm{X}$-axis of the graph designates a single probe. As you move the cursor horizontally across the graph, the probe data on the right half of the window change to correspond to the selected probe position.

The right half of the Hybridization window contains the found hybridizable sequences for individual probes. In the top box, the name (including position within the target), $\mathrm{T}_{\mathrm{m}}$ and sequence of the selected probe are displayed. The lower box contains the found hybridizable sequences. For each found sequence, the following is displayed:

- The GenBank entry that contains the sequence ("Locus").

- The position within the entry where the hybridizable sequence was found ("Pos").

- The $\mathrm{T}_{\mathrm{m}}$ or $\Delta \mathrm{G}$ of hybridization of the probe to the hybridizable sequence (" $\mathrm{T}_{\mathrm{m}}$ ").

- The nucleotide sequence of the hybridizable sequence. Identical nucleotides are displayed as "--, and mismatches are displayed as the actual nucleotide of the sequence ("Sequence").

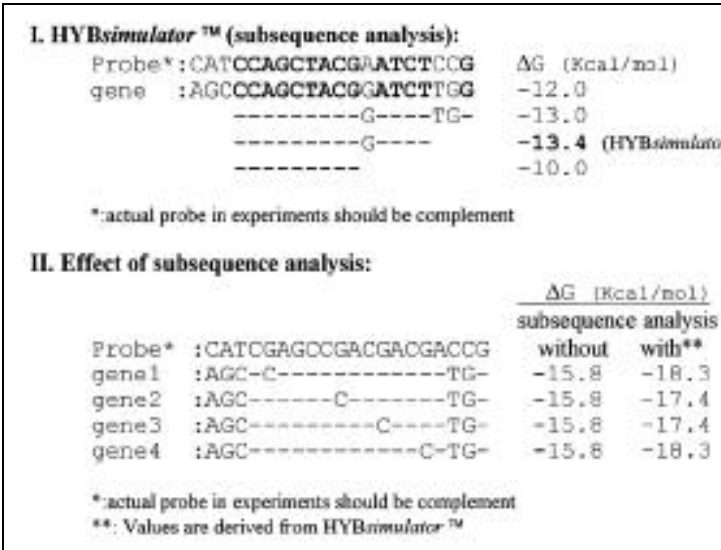

Figure 3. Comparison between conventional nearest neighbor thermodynamic calculation and HYBsimulator in mismatched nucleotide sequences.
The graph is a plot of the $T_{m}$ or $\Delta G$ of all of the found hybridizable sequences in the results file for each of the probes. The $T_{m}$ of the probe is graphed in red and will always be the highest graphed point. The $\mathrm{T}_{\mathrm{m}}$ of the first hybridization (the sequence to which the probe hybridizes with the highest $T_{m}$ ) for each probe is graphed in green. The distance from the top of the red graph to the top of the green graph indicates the difference between the $T_{m}$ of the probe and the first hybridization. More specific probes will have greater differences in these $T_{m}$ s. Since the difference of $T_{m}$ between the hybridization to the target and the first hybridization to nonspecific sequences is a measurement of a probe's specificity, we refer to this value as "Specificity". Probe P0041, indicated in Figure $4 \mathrm{~A}$, is the most specific probe of those in the graph since it has the highest Specificity. The third hybridization $\mathrm{T}_{\mathrm{m}} \mathrm{s}$ are graphed in blue, and the seventh hybridization $\mathrm{T}_{\mathrm{m}} \mathrm{s}$ are graphed in yellow. If the yellow graph reaches the height of the red graph, the probe has at least 7 exact matches (Probe P0008 in Figure 4B). By scanning the graph, the user can identify areas of particular specificity or areas in which the probes are common to other sequences.

The Library window contains the sequence database that is used for CHS. The user can view information about the hybridizable genes in the Hybridization window. If the user se-

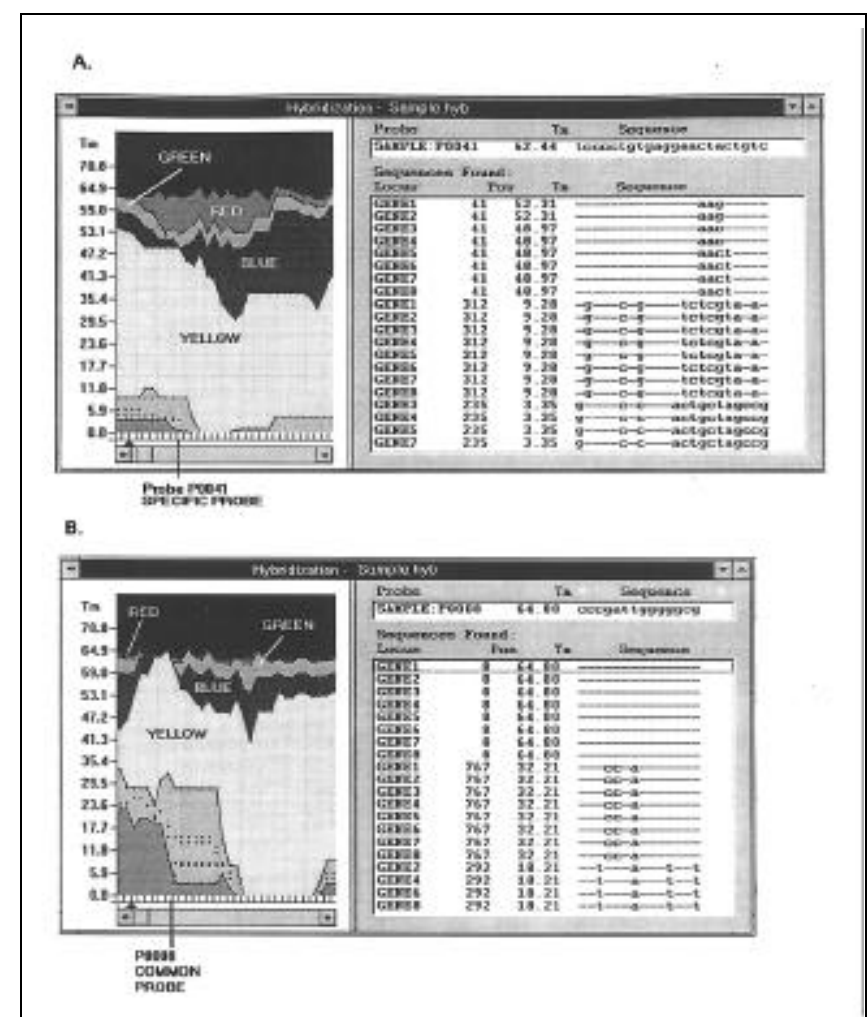

Figure 4. The Hybridization Window: identifying a specific or a common probe. Each X-axis position in the graph represents a different probe. Moving the cursor across the graph causes the data window on the right of the graph to display the data for the selected probe. (A) Probe:P0041 is selected. For each probe, the $T_{m}$ of the probe hybridizing to its target is graphed in red. The $T_{m}$ of the first hybridization is graphed in green. The $T_{m}$ of the third hybridization is graphed in blue, and the $\mathrm{T}_{\mathrm{m}}$ of the 7th hybridization is graphed in yellow. Probe:P0041 is specific because the distance between the red graph and the green graph (i.e., the specificity) is large. (B) Probe P0008 is selected. Probe:P0008 is identified as specific because the yellow graph reaches the level of the red graph, indicating that at least 7 sequences have exact matches to Probe:P0008. The data to the right of the graph verifies that $8 \mathrm{P} 0008$ have 8 exact matches. 
lects the gene name in the right side of the CHS results window, the Library window updates to display the selected sequence. The user can then examine the sequence and read the details regarding the sequence.

\section{Spreadsheet}

The data generated by CHS can be exported into a spreadsheet format, which can be opened and analyzed with most commercially available spreadsheet programs. This format allows easy manipulation and selection of candidate oligonucleotides based on the many different parameters.

The spreadsheet created by HYBsimulator contains a row for each oligonucleotide in the ProbeSet. The columns contain data, including CHS results data, that can be used to select the best oligonucleotides.

CHS data are represented by three different methods: (i) the $\mathrm{T}_{\mathrm{m}}$ of the $1 \mathrm{st}$, 2nd, 4th, 8th etc. saved hybridizable sequences found for each probe; (ii) the number of hybridizable and cross-hybridizable sequences at various $\Delta \mathrm{T}_{\mathrm{m}}$ or $\Delta \Delta \mathrm{G}$ relative to the $\mathrm{T}_{\mathrm{m}}$ or $\Delta \mathrm{G}$ of the probe hybridizing to its target; and (iii) The difference of $\mathrm{T}_{\mathrm{m}}$ or $\Delta \mathrm{G}$ between the binding strength of the probe to its target and the closest non-exact match ("Specificity").

In addition to the CHS data, the following data are also provided for each probe: $(i)$ length: the number of nucleotides in the oligonucleotide; (ii) GC content: the percentage of guanine or cytosine in the oligonucleotide; (iii) terminal GC content: the percentage $\mathrm{G} / \mathrm{C}$ of the $3^{\prime}$ or $5^{\prime}$ end of the nucleotide.
For PCR primers, because of the weaker binding of G/C base pairs, a low G/C content on the $3^{\prime}$ terminal may provide higher specificity; (iv) hairpin energy: the free energy of formation of a hairpin structure by the oligonucleotide (in Kcal $/ \mathrm{mol}$ ). Larger negative values indicate a more stable hairpin structure, which will prevent hybridization with the target and is therefore undesirable; and $(v)$ dimer energy: the free energy of formation of a dimer by the hybridization of an oligonucleotide with the same oligonucleotide. Larger negative values indicate a more stable dimer, which will compete with the hybridization with the target and can adversely affect PCR.

In a spreadsheet program, the best oligonucleotides can be selected based on all the available data with criteria specific to the application (13). Additionally, other data, such as secondary structure of the target site or estimated frequency of the oligonucleotides based on subsequences (7), can be added to the spreadsheet for selection. Also, in most commercial spreadsheet programs like Microsoft ${ }^{\circledR}$ Excel $^{\mathrm{TM}}$, users can write macros (small programs that run within the spreadsheet program) that can further derive data from the probes, which may be useful for some specific type of application or for automated selection.

\section{APPLICATIONS OF HYBsimulator}

\section{Basic Probes}

To design optimally specific DNA probes for dot blots, 
Table 1. Example of Oligonucleotide Sequences Data

\begin{tabular}{|c|c|c|c|c|c|c|c|c|}
\hline \multirow{3}{*}{$\begin{array}{c}\text { Sequence }\left(5^{\prime}-3^{\prime}\right) \\
\Delta \text { tgcagtcatagaac }\end{array}$} & \multicolumn{3}{|c|}{$\begin{array}{c}\text { Hybridization Strength } \\
(\Delta \mathrm{G}, \mathrm{Kcal} / \mathrm{mol})\end{array}$} & \multirow{2}{*}{\multicolumn{2}{|c|}{$\begin{array}{l}\text { Hairpin Dimer } \\
\text { (Kcal/mol) }\end{array}$}} & \multicolumn{3}{|c|}{$\begin{array}{c}\text { Number of Matches in GenBank } \\
\text { Primate Database at } \Delta \Delta G\end{array}$} \\
\hline & \multirow{2}{*}{$\frac{\text { DNA:DNA }}{14.8}$} & \multirow{2}{*}{$\begin{array}{c}\text { RNA:RNA } \\
20.5\end{array}$} & \multirow{2}{*}{$\frac{\text { DNA:RNA }}{13.2}$} & & & $\leq 2$ & $\leq 3$ & $\leq 4$ \\
\hline & & & & 4.1 & -6.4 & 11 & 24 & 51 \\
\hline$\nabla$ cagtcatagaacatg & 15.8 & 20.9 & 14.2 & 5.3 & -3.0 & 1 & 1 & 2 \\
\hline
\end{tabular}

Southern blots, Northern blots, etc., the user first creates a candidate ProbeSet from the target gene sequence. After selecting the model file (either for DNA:DNA, RNA:RNA or DNA:RNA), the user specifies salt concentration, oligonucleotide concentration and finally the desired $\mathrm{T}_{\mathrm{m}}$. The ProbeSet is then created containing all possible probes with the approximate $T_{m}$ specified. Then, CHS is executed for the ProbeSet against the GenBank database of interest. For example, if the project is to identify a viral DNA sequence in a human specimen, GenBank viral and human databases would be used. When the CHS is complete, the results are saved in a spreadsheet file, and the optimal or best probes are selected from the candidate probes based on the data in the spreadsheet. Typical specifications include: $\mathrm{G} / \mathrm{C}$ content between $40 \%$ and 65\%; hairpin formation energy greater than 0.0 $\mathrm{Kcal} / \mathrm{mol}$ (more negative values indicate more hairpins); dimer formation energy above $-6.0 \mathrm{Kcal} / \mathrm{mol}$ (more negative values indicate more stable dimers); and the highest specificity possible to each of the databases.

\section{Common or Consensus Probes}

It is often desirable to detect the same gene in related organisms or related genes in the same organism. It is also sometimes desirable to try to detect unknown varieties of a gene family by using a probe that identifies all known types of a particular gene family. This can be accomplished by selecting common probes to all the targets of interest.

The design of common probes is very similar to the design of basic probes. To design common probes, in addition to the steps explained for the design of basic probes, users perform an additional CHS against a small database comprised of all the target genes. The spreadsheet data for this CHS are then pasted beside the spreadsheet for the other CHS's as described for basic probes. The user first selects for the number of exact hybridizations $\left(\Delta \mathrm{T}_{\mathrm{m}}=0\right)$ to the multiple target gene database to equal the number of target sequences. From this selection, the remaining candidates are all possible common probes. From these remaining candidates (i.e., the possible common probes), the user selects based on the remaining criteria as described for basic probes.

\section{Basic PCR}

The design of PCR primers is similar to the design of probes with the following additional considerations.

Terminal G/C content. It is common practice to select primers with a low $\mathrm{G} / \mathrm{C}$ content at the $3^{\prime}$ end (i.e., the leading edge of replication). If the $3^{\prime}$ end of the primer is $\mathrm{A} / \mathrm{T}$ rich, the weaker binding of $\mathrm{A} / \mathrm{T}$ base pairs may put more importance on complete hybridization of the primer to prime polymerization. A low G/C content on the $3^{\prime}$ end of a PCR primer will therefore increase the specificity of the PCR.

Alternate binding within the target gene. It is important to ensure that primers are not going to bind at alternate sites within the target sequence. Such alternate binding can interfere with elongation or cause aberrant-sized bands. HYBsimulator can determine the most stable alternate site binding energy for each primer. Primers can be selected for minimum hybridization to alternate sites within the target.

Compatibility of primers. PCR requires two primers, and the two primers should be compatible. Compatibility of PCR primers means that both primers have very similar $\mathrm{T}_{\mathrm{m}} \mathrm{s}$, and they do not hybridize with each other. If the $\mathrm{T}_{\mathrm{m}} \mathrm{s}$ are different, at any chosen annealing temperature, either one of the primers is not annealing or the other primer is annealing to nonspecific sequences by allowing mismatches. If the primers are taken from a ProbeSet created with a fixed $\mathrm{T}_{\mathrm{m}}$, then the two primers will have the same $\mathrm{T}_{\mathrm{m}}$.

To select compatible primer pairs, the user first selects several good sense primers and then several good antisense primers. Typical criteria for selection include: G/C content between $40 \%$ and $65 \%$; hairpin formation energy greater than $0.0 \mathrm{Kcal} / \mathrm{mol}$ (more negative values indicate more hairpins); dimer formation energy above $-6.0 \mathrm{Kcal} / \mathrm{mol}$ (more negative

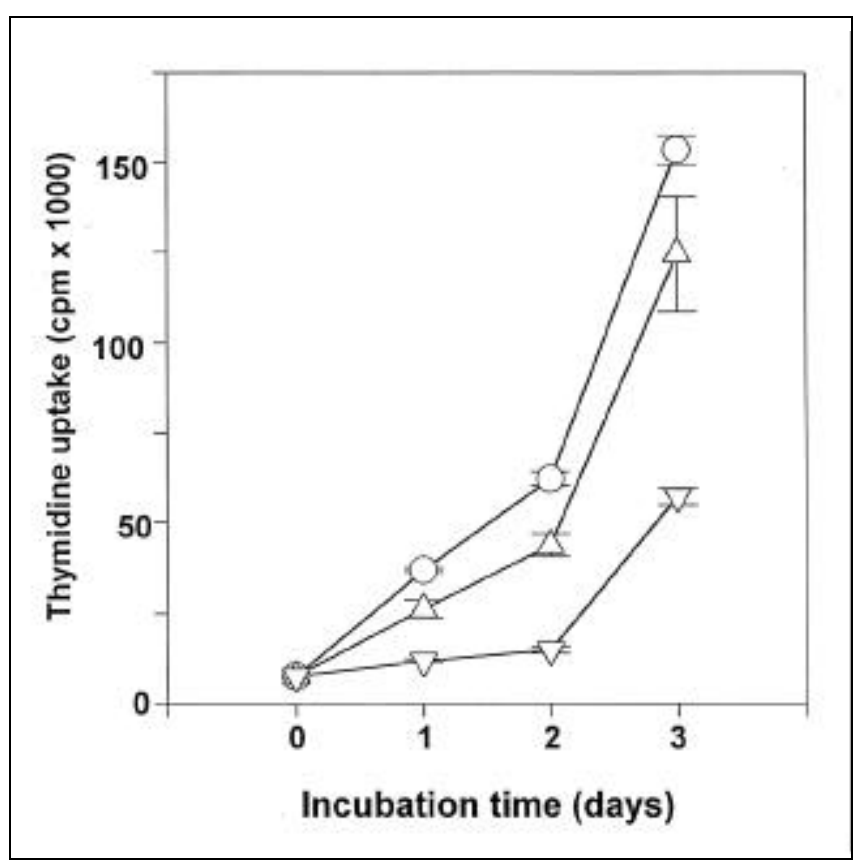

Figure 5. Inhibition of cell growth by antisense oligonucleotides. Human U937 mononuclear cells were seeded at a density of 2500 cells per well in $100 \mu \mathrm{L}$ RPMI containing $0.5 \%$ fetal calf serum and incubated with $(\Delta, \nabla)$ or without (O) $10 \mu \mathrm{M}$ of antisense phosphorothioate oligonucleotides against the human c-jun oncogene. After $24 \mathrm{~h}$ of incubation, cells were stimulated with $10 \%$ serum for $1-3$ days. Cells were exposed to ${ }^{3} \mathrm{H}$-thymidine $(0.5 \mu \mathrm{Ci}$ per well) for the last $6 \mathrm{~h}$, then incorporated radioactivity was determined in a liquid scintillation counter. Each data point is the mean \pm standard deviation from triplicate determinations. Two oligonucleotide sequences [low specificity $(\Delta)$ and high specificity $(\nabla)$ ] were selected by HYBsimulator from the start codon region as shown in Table 1. 
values indicate more stable dimers); $3^{\prime}$ end G/C content below $50 \%$; minimum hybridization to alternate sites within the target sequence and the highest specificity possible to each of the databases. From the selected sense and antisense primers, HYBsimulator will then calculate the free energy of heterodimer formation between each combination of sense with antisense primer, and the best PCR primer pair is chosen based on minimum interaction between primers.

\section{Antisense Oligonucleotides}

For antisense oligonucleotide sequence design, in addition to the basic oligonucleotide characteristics of G/C content, length and hairpin energy, and the specificity of the oligonucleotide with respect to the database, secondary structure must also be considered. Unlike hybridization experiments, the conditions of an antisense experiment cannot be changed, as they are the intracellular conditions. Therefore, more attention should be paid to the secondary structures of both the oligonucleotide and the target gene. HYBsimulator can calculate the most stable free energy of secondary structure for the target site of each probe and add the data to the spreadsheet output. Based on this information, oligonucleotides can be chosen that will hybridize outside a region containing secondary structure.

The specificity of the oligonucleotides is crucial for effective antisense inhibition. It has been reported that 9-mer phosphorothioate antisense oligonucleotides can inhibit gene function effectively and specifically when microinjected into oocytes (4). Although 9-mer oligonucleotides can be effective antisense agents, a 9-mer is more likely to hybridize to unrelated genes; therefore, extra care must be taken to select specific oligonucleotides. HYBsimulator can select candidate oligonucleotides not only by minimizing cross-hybridization against a genomic database in GenBank, but also by placing more emphasis on hybridizability against an mRNA or coding sequence database extracted from GenBank.

Figure 5 shows the results of an antisense experiment with two different antisense oligonucleotides to the c-jun oncogene and the data for the oligonucleotides.

\section{DISCUSSION}

There is no universal way to design the best oligonucleotide sequences, and design philosophies are varied for different applications. Therefore, we developed HYBsimulator to provide as much information as possible on each candidate oligonucleotide. In addition to basic oligonucleotide characteristics such as $\mathrm{G} / \mathrm{C}$ content, hairpin formation energy and dimer formation energy, we provide a quantitative means to select for specific oligonucleotides with respect to a particular database. Because of the simple spreadsheet format of the output of HYBsimulator data, users can easily add additional data that may be important for their particular application. Furthermore, because of the flexibility of HYBsimulator to use different thermodynamic models and sequence libraries, it will be useful as sequence information grows and 
additional thermodynamic nearest-neighbor parameters are developed.

One limitation of CHS is the incompleteness of current GenBank sequence data. Clearly, as GenBank becomes more complete, the correlation of CHS to the actual hybridization experiment will become closer. We believe, though, that the existing sequence data are a valid representation of the complete genome. Therefore, specificity of oligonucleotides with respect to the existing data can be a useful indication of the specificity of the oligonucleotide in the actual experiment. Moreover, if the users know particular sequences that they specifically do not want their oligonucleotide to hybridize to, CHS data will closely represent the actual experiment.

The HYBsimulator runs on an IBM compatible 486 or higher (with Windows NT ${ }^{\mathrm{TM}}$ ) or a Power Macintosh with 16-32 Mbytes of RAM, depending on the application. Because of the increasing popularity of these personal computers, we believe that HYBsimulator will be accepted as a standard tool for designing oligonucleotides in the future.

\section{ACKNOWLEDGMENTS}

We would like to thank Dr. J. SantaLucia (Wayne State University, Detroit, MI) for providing new nearest-neighbor coefficients to us, and Dr. D.G. Payan (Khepri Pharmaceuticals, South San Francisco, CA) for his critical review of the manuscript.

\section{REFERENCES}

1.Aboul-ela, J., D. Koh, I. Tinoco and F.H. Martin. 1985. Base-base mismatches. Thermodynamics of double helix formation for dCA3XA3G + cDT3YT3G (X,Y=ACGT). Nucleic Acid Res. 13:4811-4824.

2.Altschul, S.F., W. Gish, W. Miller, E.W. Myers and D.J. Lipman. 1990. Basic local alignment search tool. J. Mol. Biol. 215:403-410.

3.Breslauer, K.J., R. Frank, H. Blocker and L. Marky. 1986. Predicting DNA duplex stability from the base sequence. Proc. Natl. Acad. Sci. USA 83:3746-3750

4.Fakler, B., S. Herlitze, B. Amthor, H.P. Zenner and J.P. Ruppersberg. 1994. Short antisense oligonucleotide-mediated inhibition is strongly dependent on oligo length and concentration but almost independent of location of the target sequence. J. Biol. Chem. 269:16187-16194.

5.Feng, D.F. and R.F. Doolittle. 1987. Progressive sequence alignment as a prerequisite to correct phylogenetic trees. J. Mol. Evol. 25:351-360.

6.Freir, S.M., R. Kierzek, J.A. Jaeger, N. Sugimoto, M.H. Caruthers, T. Neilson and D.H. Turner. 1986. Improved free-energy parameters for predictions of RNA duplex. Biochemistry 83:9373-9377.

7.Han, J., Z. Zhu, C. Hsu and W.H. Finley. Selection of antisense oligonucleotides on the basis of genomic frequency of the target sequence. Antisense Res. Dev. 4:53-65.

8.He, L., R. Kierzek, J. SantaLucia, A.E. Walter and D.H. Turner. Nearest-neighbor parameters for G-U mismatches. Biochemistry 30:11241132.

9.Higgins, D.G. and P.M. Sharp. 1988. CLUSTAL: a package for performing multiple sequence alignment on a microcomputer. Gene 73:237-244.

10.Landau, G.M., U. Vishkin and R. Nussinov. 1990. Fast alignment of DNA and protein sequences. Methods Enzymol. 183:487-502.

11.Lowe, T., J. Sharefkin, S.Q. Yang and C.W. Dieffenbach. 1990. A computer program for selection of oligonucleotide primers for polymerase chain reactions. Nucleic Acids Res. 18:1757-1761.

12.Marizel, J.V. and R.P. Lenk. 1981. Enhanced graphic matrix analysis of nucleic acid and protein sequences. Proc. Natl. Acad. Sci. USA 78:76657669.

13.Mitsuhashi, M. Technical Report: oligonucleotide probe design series: basic requirements for designing optimal oligonucleotide probe sequences. J. Clin. Lab. Anal. (In press).

14.Mitsuhashi, M., A. Cooper, M. Ogura, T. Shinagawa, K. Yano and T. Hosokawa. 1994. Oligonucleotide probe design-a new approach. Na- ture 367:759-761.

15.Mitsuhashi, M. and T. Hosokawa. 1994. Novel computerized method for designing nucleotide sequence used for DNA probes and PCR primers. Nippon Rinsho 52:530-541.

16.Montpetit, M.L., S. Cassol, T. Salas and M.V. O'Shaughnessy. 1992. OLIGSCAN: a computer program to assist in the design of PCR primers homologous to multiple DNA sequences. J. Virol. Methods 36:119-128.

17.Pearson, W.R. and D.J. Lipman. 1988. Improved tools for biological sequence analysis. Proc. Natl. Acad. Sci. USA 85:2444-2448.

18.SantaLucia, J., R. Kierzek and D.H. Turner. 1990. Effects of GA mismatches on the structure and thermodynamics of RNA internal loops. Biochemistry 29:8813-8819.

19.Smith, T.F. and M.S. Waterman. 1981. Comparison of bio-sequences. Adv. Appl. Math. 2:482-489.

20.Sugimoto, N., R. Kierzek, S.M. Freier and D.H. Turner. 1986. Energetics of internal GC mismatches in ribooligonucleotide helix. Biochemistry 25:5755-5759.

21.Sugimoto, N., S. Nakano, M. Katoh, A. Matsumura, H. Nakamuta, T. Ohmichi, M. Yoneyama and M. Sasaki. 1995. Thermodynamic parameters to predict stability of RNA/DNA hybrid duplexes. Biochemistry 34:11211-11216.

22.Turner, D.H., N. Sugimoto and S.M. Freier. 1988. RNA structure prediction. Annu. Rev. Biophys. Chem. 17:167-192.

23.Wilbur, W.J. and D.J. Lipman. 1983. Rapid similarity searches of nucleic acid and protein data banks. Proc. Natl. Acad. Sci. USA 80:726-730.

Address correspondence to Masato Mitsuhashi, Hitachi Chemical Research Center, Inc., Division of Medical Sciences, Plumwood House (Univ. Cal.), 1003 Health Sciences Rd., W., Irvine, CA 92715, USA. Internet: probes@kaiwan. com 